\title{
Cellular Mechanisms and Behavioral Consequences of Kv1.2 Regulation in the Rat Cerebellum
}

\author{
Michael R. Williams, ${ }^{1}$ Jason R. Fuchs, ${ }^{3}$ John T. Green, ${ }^{3}$ and Anthony D. Morielli ${ }^{2}$ \\ ${ }^{1}$ Neuroscience Graduate Program, ${ }^{2}$ Department of Pharmacology, and ${ }^{3}$ Department of Psychology, University of Vermont, Burlington, Vermont 05405
}

\begin{abstract}
The potassium channel Kv1.2 $\alpha$-subunit is expressed in cerebellar Purkinje cell (PC) dendrites where its pharmacological inhibition increases excitability (Khavandgar et al., 2005). Kv1.2 is also expressed in cerebellar basket cell (BC) axon terminals (Sheng et al., 1994), where its blockade increases BC inhibition of PCs (Southan and Robertson, 1998a). Secretin receptors are also expressed both in PC dendrites and BC axon terminals (for review, see (Yuan et al., 2011). The effect of secretin on PC excitability is not yet known, but, like Kv1.2 inhibitors, secretin potently increases inhibitory input to PCs (Yung et al., 2001). This suggests secretin may act in part by suppressing Kv1.2. Receptor-mediated endocytosis is a mechanism of Kv1.2 suppression (Nesti et al., 2004). This process can be regulated by protein kinase A (PKA) (Connors et al., 2008). Since secretin receptors activate PKA (Wessels-Reiker et al., 1993), we tested the hypothesis that secretin regulates Kv1.2 trafficking in the cerebellum. Using cell-surface protein biotinylation of rat cerebellar slices, we found secretin decreased cell-surface Kv1.2 levels by modulating Kv1.2 endocytic trafficking. This effect was mimicked by activating adenylate cyclase (AC) with forskolin, and was blocked by pharmacological inhibitors of AC or PKA. Imaging studies identified the BC axon terminal and PC dendrites as loci of AC-dependent Kv1.2 trafficking. The physiological significance of secretin-regulated Kv1.2 endocytosis is supported by our finding that infusion into the cerebellar cortex of either the Kv1.2 inhibitor tityustoxin-K $\alpha$, or of the Kv1.2 regulator secretin, significantly enhances acquisition of eyeblink conditioning in rats.
\end{abstract}

\section{Introduction}

$\mathrm{Kv} 1.2$ is an $\alpha$-subunit of voltage-gated potassium channels that provide outward rectifying currents in diverse cell types. In the brain, Kv1.2 is abundantly expressed in the cerebellar cortex (Wang et al., 1994; Chung et al., 2001) where it strongly affects Purkinje cell (PC) activity. Kv1.2 influences excitatory synaptic input to PCs by opposing their dendritic excitability, most likely through its effect on membrane potential (Khavandgar et al., 2005; McKay et al., 2005). Kv1.2 also affects inhibitory synaptic input to PCs; pharmacological blockade of Kv1.2 in axon terminals of basket cells (BC) increases inhibitory postsynaptic currents (IPSCs) recorded in PCs. This occurs in part by removing a shunting pathway normally provided by Kv1.2 in BC axon terminals (Laube et al., 1996; Southan and Robertson, 1998a,b).

Since Kv1.2 affects excitation and inhibition of PCs, and since PCs are the sole output of the cerebellar cortex, changes in Kv1.2 functional abundance would be expected to affect cerebellar-

\footnotetext{
Received Dec. 29, 2011; revised May 17, 2012; accepted May 21, 2012.

Author contributions: M.R.W., J.R.F., J.T.G., and A.D.M. designed research; M.R.W., J.R.F., and A.D.M. performed research; M.R.W., J.R.F., J.T.G., and A.D.M. analyzed data; M.R.W., J.R.F., J.T.G., and A.D.M. wrote the paper.

This work was supported in part by National Institutes of Health (NIH) Grant 5 P20 RR016435-07 from the Center of Biomedical Research Excellence Program of the National Center of Research Resources, NIH Grant RONS050623 (to A.D.M.), and the University of Vermont Department of Psychology McNeil Prevention and Community Psychology Fund (to J.T.G.). We thank Victor May and Deborah Damon for feedback on the manuscript and Gain M. Robinson for analysis of cannula placements.

The authors declare no competing financial interests.

Correspondence should be addressed to Anthony D. Morielli, University of Vermont, College of Medicine, Department of Pharmacology, 89 Beaumont Avenue, Given Building Room E 317, Burlington, VT 05403. E-mail: anthony.morielli@uvm.edu.

DOI:10.1523/JNEUROSCI.6504-11.2012

Copyright $\odot 2012$ the authors $\quad 0270-6474 / 12 / 329228-10 \$ 15.00 / 0$
}

dependent behaviors. This idea is supported by the identification of a mutation within Kv1.2 that reduces the channel's surface expression and causes cerebellar ataxia in mice (Xie et al., 2010). In HEK293 cells, surface expression and electrophysiological function of Kv1.2 is regulated by its endocytic trafficking (Nesti et al., 2004). In this heterologous expression system, Kv1.2 trafficking is regulated by adenylate cyclase (AC) and protein kinase A (PKA) (Connors et al., 2008), raising the possibility that ACdependent trafficking of Kv1.2 occurs in the cerebellum. The Gs-coupled secretin receptor is a candidate for AC-mediated regulation of cerebellar Kv1.2 because, like Kv1.2, it is expressed in PCs and BC axon terminals (for review, see Yuan et al., 2011). There is functional support for this idea, as application of secretin to rat cerebellar slices increases IPSC activity recorded from PCs (Yung et al., 2001), a phenomenon that can also be evoked by pharmacological inhibition of Kv1.2 in BC axon terminals (Southan and Robertson, 1998a,b). Last, while the downstream targets of secretin-stimulated AC are not well characterized, the regulation of presynaptic potassium conductance has been raised as a potential mechanism (Yung et al., 2001). We therefore tested the hypothesis that cerebellar Kv1.2 is regulated by secretin.

Here we report that secretin decreases cell-surface expression of cerebellar Kv1.2 through a process involving AC/PKAdependent channel endocytosis. We provide evidence that Kv1.2 trafficking occurs in the pinceaus of $\mathrm{BC}$ axon terminals and in PC dendrites. A functional role for this regulation is supported by our finding that infusion of tityustoxin-K $\alpha$ (TsTx) into the cerebellar cortex enhances eyeblink conditioning (EBC) and that infusion of secretin has the same effect, likely through the net inhibition of PC output. Together our data support the hypoth- 
esis that secretin regulates Kv1.2 in vivo and that this regulation is behaviorally significant.

\section{Materials and Methods}

Generation of cerebellar slices. Parasagittal 250- to 400- $\mu$ m-thick cerebellar sections were generated from 3- to 6-week-old male Sprague Dawley rats using a Leica VT1000S vibratome. Dissection and slice generation was in ice-cold, modified artificial CSF (ACSF) in which $\mathrm{NaCl}$ was replaced by sucrose containing the following (in $\mathrm{mM}$ ): 250 sucrose, 26 $\mathrm{NaHCO}_{3}, 2.5 \mathrm{KCl}, 3 \mathrm{MgCl}_{2}, 1 \mathrm{CaCl}_{2}, 10$ glucose, $1.25 \mathrm{NaH}_{2} \mathrm{PO}_{4}, 0.4$ and sodium ascorbate, gassed with $95 \% \mathrm{O}_{2}, 5 \% \mathrm{CO}_{2}$ ) (Moyer and Brown, 1998; Southan and Robertson, 1998a). After generation, slices were briefly maintained in room temperature ACSF containing the following (in mM): $125 \mathrm{NaCl}, 26 \mathrm{NaHCO}_{3}, 2.5 \mathrm{KCl}, 2 \mathrm{MgCl}_{2}, 2 \mathrm{CaCl}_{2}, 10$ glucose, $1.25 \mathrm{NaH}_{2} \mathrm{PO}$, and 0.4 sodium ascorbate, gassed with $95 \% \mathrm{O}_{2}, 5 \% \mathrm{CO}_{2}$ ) until transferred to interface culture (De Simoni and Yu, 2006). Pharmacological agents were added directly to culture media after slices had recovered in culture for at least an hour. Slices were cultured and exposed to pharmacological manipulations individually. For biotinylation assays (except the gabazine series), and for the imaging studies, slices were pretreated with $1 \mu \mathrm{M}$ tetrodotoxin (EMD Chemicals) to prevent effects from altered sodium action-potential activity by the other drug treatments.

Biotinylation of surface proteins. After drug treatments, cerebellar slices were individually biotinylated with $1 \mathrm{ml}$ of sulfo-nhs-ss-biotin ("biotin") (Pierce) at $2 \mathrm{mg} / \mathrm{ml}$ in ice-cold HBSS, a concentration we determined sufficient to recover all Kv1.2 from a permeabilized slice. Unreacted biotin was quenched with $50 \mathrm{~mm}$ TRIS. For internalization assays, slices were biotinylated, quenched, and then returned to interface culture for pharmacological treatment, after which remaining surface biotinylated proteins were cleaved by two 20 min incubations in ice-cold $50 \mathrm{~mm}$ glutathione, $75 \mathrm{~mm} \mathrm{NaCl}, 10 \mathrm{~mm}$ EDTA, $0.1 \%$ bovine serum albumin, and $0.075 \mathrm{~N} \mathrm{NaOH}$ (Yang et al., 2005). In the indicated experiments of Figure 4 , slices were extracted in acetone after biotinylation and quenching, before lysate generation.

Preparation of biochemical samples. Biotinylated cerebellar slices were individually sonicated in lysis buffer [50 mM TRIS, $150 \mathrm{~mm} \mathrm{NaCl}, 1 \mathrm{~mm}$ EDTA, $0.25 \%$ deoxycholate, $1 \%$ NP40, 10\% glycerol, and mammalian protease inhibitor cocktail (Sigma), $\mathrm{pH}$ 8.0], and the lysate was then clarified by centrifugation. A portion of clarified "Total" lysate was reserved and the remainder was incubated with high-capacity neutravidin bead slurry (Pierce). Neutravidin beads and bound biotinylated proteins were isolated by centrifugation and washed repeatedly with lysis buffer. In some experiments, a portion of the post-neutravidin supernatant lysate was analyzed as a "Post" sample. Biotinylated proteins were eluted from neutravidin beads by incubation at $100^{\circ} \mathrm{C}$ in Laemmli sample buffer containing $0.1 \mathrm{~m}$ dithiothreitol (DTT) (Sigma) to produce the "Eluant." Total and Post lysate samples were similarly prepared for immunoblot by incubation at $100^{\circ} \mathrm{C}$ in sample buffer and DTT.

Biotinylation sample analysis. For each slice, Total and Eluant samples were resolved by PAGE and protein immunoblot as previously published (Williams et al., 2007). Primary antibodies were mouse monoclonal $\alpha$-Kv1.2 (K14/16; Neuromab) and rabbit polyclonal $\alpha$-glyceraldehyde3-phosphate dehydrogenase (GAPDH) (ab9485; Abcam).

According to the supplier, the $\alpha$-Kv1.2 antibody immunogen is rat Kv1.2, giving no reported reactivity toward Kv1.1, Kv1.3, Kv1.4, Kv1.5, or Kv1.6, or toward brain-derived samples from the Kv1.2 knock-out mouse.

Infrared secondary antibody signals were detected and quantified using an Odyssey Infrared Imager (LI-COR). Surface Kv1.2 was calculated by dividing the density of Kv1.2 signal in the Eluant by the density of Kv1.2 signal in the Total for that slice. Total Kv1.2 was determined by dividing the density of Kv1.2 signal in the Total by the density of GAPDH signal in the same Total sample. The proportion of Kv1.2 bound to neutravidin was calculated by dividing the density of Kv1.2 signal in the Post fraction by that in the Total fraction, normalized to the ratio from a nonbiotinylated sample in the same experiment to control for nonspecific loss.

Biotinylation sample statistics. Within an experiment, Surface and Total Kv1.2 values were normalized to the average of vehicle-treated slices.
Each experiment was repeated using slices derived from multiple rats, and normalized values from individual experiments were pooled for analysis. Because each slice was cultured, drug treated, biotinylated, lysed, and evaluated by Western blot individually, " $n$ " is the total number of slices from all animals per pharmacological condition. The $n$ for each condition is identified in the text as " $n$ (vehicle), $n$ (treatment); from $x$ rats)." Effects of pharmacological manipulations are expressed graphically and in text as the mean percentage change relative to vehicle. In graphs, error bars indicate SEM. Statistical significance is indicated by an asterisk $\left(^{*}\right)$, defined as a $p$ value $\leq 0.05$ by unpaired $t$ test. Welch's correction was used if the variance was unequal between control and stimulus groups (Ruxton, 2006).

ATTO 594-TsTx. A linear 37 mer peptide for TsTx (ATTO-TsTx) with an alanine to cysteine substitution at site 20 was synthesized by CelTek Bioscience. Cysteine-directed conjugation to the fluorescent dye ATTO 594, refolding, purification, and validation of toxin activity by electrophysiology on rat Kv1.2 expressed in Xenopus oocytes was performed by Alomone Labs.

ATTO-TsTx microscopy. Rat cerebellar slices that were vehicle or forskolin treated as above were gently rinsed in ice-cold HBSS before fixation on ice in $4 \%$ formaldehyde in HBSS. Slices were incubated overnight in 3 nм ATTO-TsTx, rinsed, and postfixed. Slices were mounted with Prolong Gold anti-fade (Invitrogen) and imaged using the DeltaVision Restoration Microscopy System (Applied Precision). Images were processed with ImageJ software. Parallel experiments compared $\alpha-\operatorname{Kv} \beta 2$ (Neuromab 75-021) labeling in slices fixed under the same conditions, followed with or without permeabilization by ice-cold acetone. Positive $\mathrm{Kv} \beta 2$ staining was compared with IgG control, detected by an Alexa 647-conjugated secondary antibody (Invitrogen).

Kv1.2 immunofluorescence. Rat cerebellar slices that were vehicle or forskolin treated as above were gently rinsed in ice-cold HBSS, then extracted with ice-cold acetone before fixation in $4 \%$ formaldehyde in HBSS. Slices were then incubated in blocking buffer (10\% normal goat serum in PBS), then in primary and secondary antibodies. Primary antibodies were mouse monoclonal $\alpha$-Kv1.2 (Neuromab) or isotypematched mouse IgG. The secondary antibody was goat anti-mouse Alexa 568 (Invitrogen). Slices were mounted with Prolong Gold anti-fade (Invitrogen) and imaged using the DeltaVision Restoration Microscopy System (Applied Precision). Images were processed with ImageJ software.

Microscopy analysis. Within distinct areas of the cerebellar cortex, multiple fields of view (FOV) were acquired. The sum projections of serial $z$-sections for each FOV were generated to collapse dimensionality. Within each FOV projection, a region of interest (ROI) was defined for the molecular layer, each pinceau, and within the granule cell layer. The mean intensity of ATTO-TsTx in granule cell ROIs was subtracted from the intensity measured in molecular layer and in pinceau ROIs to control for local variations in slice thickness and for experimental variability. For experiments using $\alpha$-Kv1.2, the background was defined by isotypematched IgG incubated slices. These background-corrected Kv1.2 intensity measurements from an individual animal in the vehicle treatment for the same area of interest (molecular layer or pinceau) were averaged together for normalization of the percentage change observed in forskolin-treated slices. Effects of pharmacological manipulations are expressed graphically and in text as the mean percentage change relative to vehicle, $n$ is the total number of ROIs from all animals per pharmacological condition per area of study. The $n$ for each condition is identified in the text as $n$ (vehicle), $n$ (treatment); from $x$ rats). Statistical significance is indicated by an asterisk $\left(^{*}\right)$, defined as a $p$ value $\leq 0.05$ by unpaired $t$ test.

Electrophysiology. Sagittal cerebellar slices generated as described above were maintained for at least $1 \mathrm{~h}$ in room temperature ACSF bubbled with carbogen in a home-made maintenance chamber before being transferred to the recording chamber. Slices were visualized using a $60 \times$ water-immersion objective and infrared differential interference contrast optics on an upright Olympus microscope. Temperature was maintained at $35^{\circ} \mathrm{C}$ and the recording chamber was perfused with carbogenated ACSF at $1.5-2 \mathrm{ml} / \mathrm{min}$. Single-cell extracellular recordings of PC action-potential activity was with a patch electrode filled with ACSF and an Axopatch 200D amplifier interfaced via a DigiData 1322A 
D/A converter to a Windows-based computer running the pCLAMP data acquisition program Clampex (Molecular Devices). Data analysis was performed with the PCLAMP data analysis program Clampfit (Molecular Devices) and with Minianalysis (Synaptosoft). Action-potential activity was measured in a 1 min window before secretin (30 nM) and compared in a $1 \mathrm{~min}$ window starting at $15 \mathrm{~min}$ after secretin addition. Each reported recording was from a PC in individual slices derived from six animals.

EBC subjects. Subjects were 30 male Wistar rats from Harlan. Rats were between 59 and $63 \mathrm{~d}$ old when they arrived in the colony. After arrival, the rats were housed individually for $\sim 1$ week before surgery with ad libitum chow and water. The colony was maintained on a $12 \mathrm{~h}$ light/dark cycle (lights on at 7:00 A.M.). All procedures were approved by the Institutional Animal Care and Use Committee at the University of Vermont.

$E B C$ surgery. Rats were anesthetized using 3\% isoflurane in oxygen and, using aseptic surgical procedures, each rat was surgically prepared with a 22 gauge guide cannula that was implanted in the left cerebellar cortex (lobulus simplex), ipsilateral to the conditioned eye, at the following coordinates: -11.3 anteroposterior (from bregma), +2.5 mediolateral, and -3.1 dorsoventral. Subsequently, rats were fitted with differential electromyographic (EMG) recording wires for recording eyeblinks and a bipolar periocular stimulation electrode for delivering the eye stimulation unconditioned stimulus (US). The EMG wires for recording activity of the external muscles of the eyelid, the orbicularis oculi, were constructed of two strands of ultrathin $(75 \mu \mathrm{m})$ Teflon-coated stainless steel wire soldered at one end to a mini-strip connector. The other end of each wire was passed subdermally to penetrate the skin of the upper eyelid of the left eye and a small amount of the insulation was removed. The bipolar stimulation electrode (Plastics One) was positioned subdermally immediately dorsocaudal to the left eye. The ministrip connector and the bipolar stimulation electrode were adhered to the skull with dental cement. In addition, a ground wire was connected to three stainless steel skull screws. The wound was salved with antibiotic ointment (povidone), and an analgesic (buprenorphine) was administered subcutaneously immediately after surgery and twice the following day. Rats were given a minimum of $5 \mathrm{~d}$ to recover before EBC.

$E B C$ apparatus. EBC took place in one of four identical testing chambers $(30.5 \times 24.1 \times 29.2 \mathrm{~cm}$; Med Associates), each with a grid floor. The top of each chamber was modified so that a 25 -channel tether/commutator could be mounted to it. Each testing chamber was housed within an electrically shielded, sound-attenuating chamber $(45.7 \times 91.4 \times 50.8$ $\mathrm{cm}$; BRS-LVE). A fan in each sound-attenuating chamber provided background noise of $\sim 60 \mathrm{~dB}$ sound pressure level. A speaker was mounted in each corner of the rear wall and a house light (off during testing) was mounted in the center of the rear wall of each sound-attenuating chamber. The sound-attenuating chambers were housed within a walk-in sound-proof chamber.

Stimulus delivery and recording of eyelid EMG activity were controlled by a computer interfaced with a Power 1401 high-speed data acquisition unit and running Spike2 software (CED). A $2.8 \mathrm{kHz}, 80 \mathrm{~dB}$ tone $(765 \mathrm{~ms}$ for the TsTx experiment; $865 \mathrm{~ms}$ for the secretin experiment) served as the conditioned stimulus (CS). A $15 \mathrm{~ms}, 4.0 \mathrm{~mA}$ unipolar periocular stimulation, delivered from a constant current stimulator (model A365D; World Precision Instruments), served as the US. The eyelid EMG signals were amplified (10 k) and bandpass filtered (100$1000 \mathrm{~Hz}$ ) before being passed to the Power 1401 and from there to a computer running Spike2 (CED). Spike2 was used to full-wave rectify, smooth (10 ms time constant), and time shift ( $10 \mathrm{~ms}$, to compensate for smoothing) the amplified EMG signal.

$E B C$ procedure. At the beginning of each session, each rat's cemented connector was attached to the 25-channel tether/commutator, which carried leads to and from peripheral equipment and allowed the rat to move freely within the testing box. On day 1 (adaptation), rats were plugged in but no stimuli were delivered. They remained in the chamber for $60 \mathrm{~min}$ (the approximate length of a training session). Spontaneous eyelid EMG activity was sampled for the same duration and at the same time points as during the subsequent conditioning sessions. On days $2-8$ (conditioning), rats received 100 trials per day, with an average intertrial interval of $30 \mathrm{~s}$ (range $=20-40 \mathrm{~s})$. Each block of 10 trials consisted of the following trial sequence: four CS-US trials (CS preceding and coterminating with the US), one CS-alone trial, four CS-US trials, and one US-alone trial.

Two separate behavioral experiments were conducted. In the first experiment, immediately before the first $6 \mathrm{~d}$ of conditioning, rats underwent an infusion of $1 \mu \mathrm{l}$ of either $0.25 \mu \mathrm{g}$ TsTx or PBS vehicle. In the second experiment, immediately before the first $3 \mathrm{~d}$ of conditioning, rats underwent an infusion of $1 \mu \mathrm{l}$ of either $1.0 \mu \mathrm{g}$ secretin or PBS vehicle. For infusions, the dummy cannula was removed and a 28 gauge internal cannula was inserted into the guide cannula. The internal cannula protruded $1 \mathrm{~mm}$ below the guide cannula tip; thus, infusions were delivered at $4.1 \mathrm{~mm}$ below bregma. Infusions were made with a $10 \mu \mathrm{l}$ Hamilton syringe loaded onto an infusion pump (KD Scientific) set to deliver $1 \mu \mathrm{l}$ over a period of $2 \mathrm{~min}$. At the end of the infusion period, the internal cannula remained in place for an additional $1 \mathrm{~min}$ to allow diffusion of the solution away from the cannula tip. Subsequently, the internal cannula was removed, the dummy cannula was replaced, and the conditioning procedure began. Rats were infused and tested in groups of four (two from each group).

Histology for cannula placements. Following the last day of training, rats were overdosed with sodium pentobarbital $(150 \mathrm{mg} / \mathrm{kg})$ and transcardially perfused with $0.9 \%$ saline followed by $10 \%$ formalin. A small amount of direct current $(100 \mu \mathrm{A}$ for $10 \mathrm{~s})$ was passed through an insulated 00 ( $0.3 \mathrm{~mm}$ diameter) stainless steel insect pin inserted into the cannula so that the tip of the insect pin, which was uninsulated, protruded $\sim 1 \mathrm{~mm}$ below the guide cannula tip. Subsequently, the brain was removed and stored in $10 \%$ formalin.

Approximately $3 \mathrm{~d}$ before sectioning, brains were transferred to a $30 \%$ sucrose $/ 10 \%$ formalin solution. On the day of sectioning, cerebella were embedded in albumin-gelatin, frozen, and $60 \mu \mathrm{m}$ coronal sections were taken with a cryostat through areas with cannula placements. The tissue was mounted on gelatin-subbed slides and stained with cresyl violet (for cell bodies) and Prussian blue (for iron deposits from the marking lesions). Slides were coverslipped with Permount and examined under a microscope by an observer blind to group membership to confirm cannula placement.

$E B C$ analysis. CS-US trials were subdivided into three time periods: (1) a "baseline" period, $280 \mathrm{~ms}$ before CS onset; (2) a "startle" period, 0-80 ms after CS onset; and (3) a conditioned response (CR) period, 81-750 ms (TsTx experiment) or 81-850 ms (secretin experiment) after CS onset. Eyeblinks that exceeded mean baseline activity by 0.5 A.U. (range $=0.0-5.0$ ) during the CR period were scored as CRs. This time point was also defined as CR onset. The difference in time (in milliseconds) between CS onset and CR onset represents CR onset latency. The maximum eyelid closure during the CR was CR amplitude. Eyeblinks that met the response threshold during the startle period were scored as startle responses (SRs). UR (unconditioned response) amplitude was assessed on US-alone trials $65-165$ after US onset (the first $65 \mathrm{~ms}$ was obscured by the shock artifact). Data were analyzed using SPSS 20.0.0. An $\alpha$ level of 0.05 was used as the rejection criterion for all statistical tests.

\section{Results}

Characterization of Kv1.2 trafficking in the cerebellum

Quantification of surface and internal Kv1.2 pools using a biotinylation assay

Pharmacological and genetic studies demonstrate the importance of Kv1.2 to cerebellar function. Kv1.2 electrophysiological activity is controlled by receptor-mediated regulation of its trafficking at the cell surface (Nesti et al., 2004; Williams et al., 2007; Connors et al., 2008; Stirling et al., 2009). As a first step toward examining Kv1.2 trafficking in the cerebellum, we used a biotinylation assay to quantify the proportion of total Kv1.2 at the cell surface in rat cerebellar slices. Application of the biotinylation reagent sulfo-nhs-ss-biotin (Pierce) to cerebellar slices labeled surface but not intracellular protein. By immunoblot we found that Kv1.2, but not GAPDH, was present in the Eluant from neutravidin beads incubated with lysates of biotinylated cerebellar slices, although both proteins were present in the Total lysate 
A

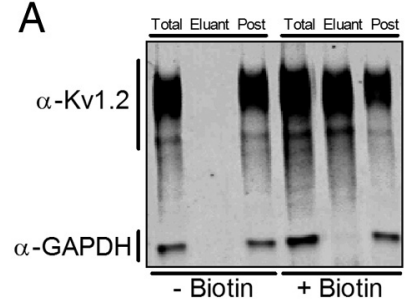

C

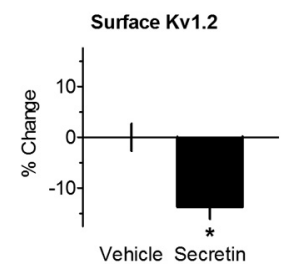

$\mathrm{D}$

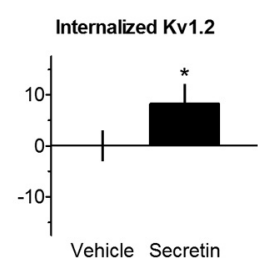

B

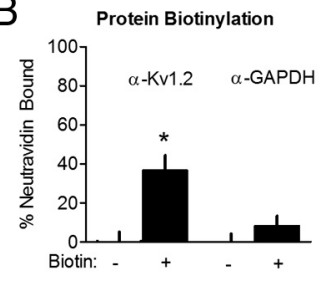

E

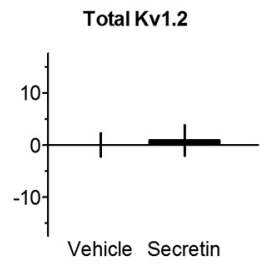

Figure 1. Secretin regulates Kv1.2 surface expression in the cerebellum. $\boldsymbol{A}$, In cerebellar slices treated with $2 \mathrm{mg} / \mathrm{ml}$ biotin (+Biotin) or not (-Biotin), both GAPDH and Kv1.2 are present in the Total lysate. Kv1.2 is present in neutravidin bead Eluant of + Biotin slices, while GAPDH is not, indicating selective biotinylation of cell-surface proteins. Kv1.2 is diminished in the Post fraction after the Total is incubated with neutravidin. $\boldsymbol{B}$, The percentage decrease in GAPDH or Kv1.2 in the Post versus Total fraction from slices biotinylated or not following incubation with neutravidin. Compared with - Biotin slices, $\sim 36 \%$ of all Kv1.2 is neutravidin bound in + Biotin slices $\left({ }^{*} p \leq 0.0006\right)$. In contrast, there is no significant difference in the amount of neutravidin-bound GAPDH between - Biotin and + Biotin slices $(p \geq 0.23)$. C, Change in mean surface Kv1.2 as determined by biotinylation and immunoblot of drug-treated cerebellar slices. A 15 min incubation in $30 \mathrm{~nm}$ secretin, relative to vehicle, reduces Kv1.2 at the cell surface in cerebellar slices $\left({ }^{*} p \leq 0.0002\right)$. In the presence of $30 \mu \mathrm{m}$ secretin receptor antagonist, $5-27$ secretin, secretin does not change the surface expression of Kv1.2 ( $p=0.93$ ). $D$, The change in mean amount of Kv1.2 internalized from the plasma membrane as determined by biotinylation and immunoblot. Compared with vehicle, secretin increases the mean internalized population of Kv1.2 ( $\left.{ }^{*} p \leq 0.05\right)$. $\boldsymbol{E}$, As determined by immunoblot of the samples in which internalization was measured, secretin does not alter mean total Kv1.2 levels relative to vehicle $(p \geq 0.82)$.

(Fig. 1A). Kv1.2 was not present in the neutravidin eluant without biotinylation, confirming that that biotinylation allowed selective recovery of cell surface Kv1.2 (Fig. 1A). Incubation with neutravidin removes biotinylated surface Kv1.2 from the Total lysate. Therefore, the abundance of Kv1.2 found in the postneutravidin Post fraction, relative to the abundance in the Total fraction, defines the measurable surface pool of Kv1.2. Determining this ratio for nonbiotinylated samples allowed normalization for nonspecific protein loss. Using this method, we found the proportion of Kv1.2 in the Post versus Total fractions was significantly different in biotinylated samples $(-36.46 \pm 9.40 \%, p=$ $0.0006, n=15,15$; three rats). By comparison, in these same samples, there was no difference in the proportion of GAPDH between these nonbiotinylated and biotinylated samples (8.04 \pm $6.58 \%, p=0.23, n=15,15$; three rats) (Fig. $1 B$ ), validating the selective labeling of cell-surface proteins. Our finding that the majority of Kv1.2 is not biotinylatable by these methods suggests the existence of regulated trafficking of Kv1.2 between the plasma membrane and intracellular compartments.

\section{Exogenous secretin regulates cell-surface Kv1.2 trafficking}

Secretin enhances inhibitory output from BCs to PCs. Pharmacological inhibition of Kv1.2 (Southan and Robertson, 1998b; Tan and Llano, 1999) mimics this effect, suggesting that secretin suppresses Kv1.2. Because suppression of Kv1.2 ionic current can be caused by channel endocytosis (Nesti et al., 2004), we measured the effect of exogenous secretin on surface Kv1.2 levels using cell-surface protein biotinylation of rat cerebellar slices

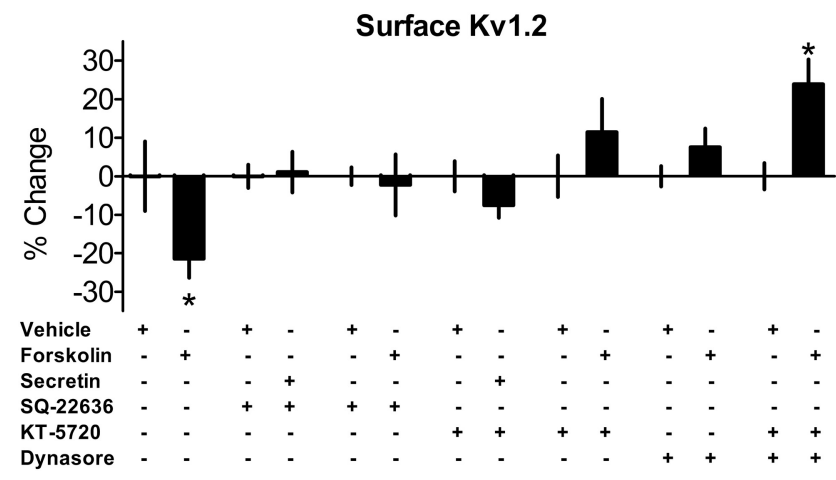

Figure 2. Secretin regulates Kv1.2 via AC/PKA. Change in mean surface Kv1.2 as determined by biotinylation and immunoblot of drug-treated cerebellar slices. $A 15$ min incubation in the $A C$ activator forskolin $(100 \mu \mathrm{M})$ reduces mean surface Kv1.2 $\left({ }^{*} p \leq 0.05\right)$. In the presence of the AC inhibitor SQ-22536 (100 $\mu \mathrm{M})$ neither secretin ( $p \geq 0.86)$ nor forskolin $(p \geq 0.78)$, relative to their vehicle, changes surface Kv1.2. In the presence of the PKA inhibitor KT-5720 (5 $\mu \mathrm{M}$ ) neither secretin ( $p \geq 0.16$ ) nor forskolin ( $p \geq 0.26$ ), relative to their vehicle, changed surface Kv1.2. The dynamin inhibitor dynasore $(80 \mu \mathrm{M})$ prevents forskolin-induced reduction in surface Kv1.2 $(p \geq 0.18)$. Under inhibition of both PKA (KT-5720,5 $\mu \mathrm{M}$ ) and dynamin (dynasore, $80 \mu \mathrm{M})$, forskolin, compared with vehicle, increases surface Kv1.2 ( $\left.{ }^{*} p \leq 0.005\right)$.

(Fig. 1C). Secretin (30 nm, $15 \mathrm{~min}$ ) (Anaspec) decreased surface Kv1.2 relative to vehicle $(-13.71 \pm 3.54 \%, p=0.0002, n=52$, $51 ; 12$ rats). In the presence of a secretin receptor antagonist, 5-27 secretin (Anaspec) $(30 \mu \mathrm{M})$, secretin had no effect $(-0.96 \pm$ $10.68 \%, p=0.93, n=12,9$; three rats). To determine whether the secretin-induced decrease in surface Kv1.2 involved endocytosis, we measured the secretin-modulated internalization of biotinylated Kv1.2 (Fig. 1D). Secretin increased the amount of internalized Kv1.2 relative to vehicle $(+8.24 \pm 4.65 \%, p=0.047$, $n=22,22$; three rats). In the same slices, total levels of Kv1.2 (Fig. $1 E)$ protein did not differ $(-0.86 \pm 3.65 \%, p=0.82, n=22,22$; three rats). Endocytic trafficking of Kv1.2 involves both endocytosis from, and recycling back to, the plasma membrane (Stirling et al., 2009). Therefore, the increased internalized pool of Kv1.2 observed in secretin-treated slices could be the result of either a net increase in Kv1.2 endocytosis from, or a net decrease in its recycling back to, the plasma membrane. While the internalization assay used here does not distinguish between these mechanisms, the results presented here demonstrate that secretin receptor signaling modulates the endocytic trafficking of Kv1.2 in the cerebellum.

\section{Secretin regulates Kv1.2 trafficking through the Gs/cAMP/} PKA pathway

Having found that secretin regulates cerebellar Kv1.2, we sought to determine the cellular mechanisms involved. Secretin receptors preferentially couple to Gs (for review, see Siu et al., 2006) and secretin enhancement of BC neurotransmission involves AC (Yung et al., 2001). Therefore, we hypothesized secretin acts via $\mathrm{AC}$ to regulate Kv1.2. In support of this, the AC activator, forskolin (100 $\mu \mathrm{M}, 15 \mathrm{~min}$; Calbiochem) reduced surface Kv1.2 $(-21.36 \pm 9.71 \%, p=0.036, n=13,17$; three rats) (Fig. 2). As with secretin, there was no corresponding change in total Kv1.2 $(+8.17 \pm 11.65 \%, p=0.48, n=16,17$; three rats). Confirming the role of AC, pretreatment with the AC inhibitor SQ-22536 (Calbiochem) $(100 \mu \mathrm{M}, 20 \mathrm{~min})$, blocked secretin's $(+1.07 \pm$ $6.08 \%, p=0.86, n=17,17$; three rats $)$ and forskolin's $(-2.26 \pm$ $8.27 \%, p=0.78, n=13,13$; four rats) effect on surface Kv1.2.

A previous study in HEK293 cells suggests PKA enhances Kv1.2 endocytosis (Connors et al., 2008). We therefore asked whether PKA was the effector of AC in the regulation of cerebellar 
Kv1.2. After pretreatment with the PKA inhibitor KT-5720 (5 $\mu \mathrm{M}, 30 \mathrm{~min})$ (Calbiochem), neither secretin $(-7.49 \pm 5.17 \%$, $p=0.16, n=15,14$, three rats $)$ nor forskolin $(-11.49 \pm 10.30 \%$, $p=0.26, n=20,21$; five rats) altered surface expression of Kv1.2. In HEK cells, Kv1.2 endocytosis is dynamin dependent (Nesti et al., 2004; Stirling et al., 2009). Consistent with this, we found that in cerebellar slices, pretreatment with the dynamin inhibitor, dynasore (Calbiochem) (80 $\mu \mathrm{M}, 30 \mathrm{~min})$, prevented the forskolin-stimulated reduction of surface $\mathrm{Kv1.2}(+7.57 \pm$ $5.50 \%, p=0.181, n=18,17$; three rats).

In contrast to PKA-dependent endocytosis, cAMP generated by AC activity in HEK293 cells can also cause a PKA-independent increase of surface Kv1.2 (Connors et al., 2008). This bidirectional modulation increases the dynamic range of surface Kv1.2 that can result from AC stimulation. Therefore we tested whether forskolin stimulation could stimulate an increase in surface Kv1.2 independent of PKA-dependent endocytosis in cerebellar slices. We found that, in cerebellar slices pretreated with both KT-5720 $(5 \mu \mathrm{M})$ and dynasore $(80 \mu \mathrm{M})$ for $30 \mathrm{~min}$, application of forskolin elicited a significant increase in surface Kv1.2 relative to vehicle control $(+23.88 \pm 7.32 \%, p=0.0032, n=18,18$; three rats) (Fig. 2 ). Thus, although the cellular mechanisms for the bidirectional regulation of surface Kv1.2 by AC exist in the cerebellum, under our experimental conditions, stimulation of the secretin receptor results predominantly in an AC-mediated PKA-dependent decrease in surface Kv1.2.

\section{Regulation of Kv1.2 by endogenous secretin}

The previous experiments demonstrate that activation of secretin receptors with exogenous secretin can regulate cerebellar Kv1.2 trafficking. We therefore asked whether endogenously released secretin regulates Kv1.2. The proposed mechanism for the endogenous activation of secretin receptors is depolarizationinduced release of secretin from PCs (Köves et al., 2002, 2004; Lee et al., 2005a,b). PC activity is normally constrained by $\mathrm{GABA}_{\mathrm{A}}$ mediated inhibition; blocking $\mathrm{GABA}_{\mathrm{A}}$ receptors can increase $\mathrm{PC}$ firing in culture (Gähwiler, 1975). Therefore, the $\mathrm{GABA}_{\mathrm{A}}$ receptor antagonist, gabazine (SR-95531; Sigma-Aldrich), could increase endogenous secretin release from depolarized PCs. We hypothesized that secretin so released would activate secretin receptors and thereby reduce surface Kv1.2. Supporting this model, gabazine $(20 \mu \mathrm{M}, 45 \mathrm{~min})$ decreased surface Kv1.2 (-20.41 \pm $6.19 \%, p=0.003, n=13,13$; three rats) (Fig. 3), without changing total $\mathrm{Kv1.2}(-6.22 \pm 7.71 \%, p=0.43, n=13,13$; three rats $)$. The mechanism by which depolarization of PCs enhances secretin release involves activation of voltage-gated calcium channels (VGCCs) (Lee et al., 2005a). Consistent with this, we found that gabazine had no effect on surface Kv1.2 in the presence of nimodipine $(20 \mu \mathrm{M})$ (Calbiochem), an L-type VGCC blocker $(-1.39 \pm 6.43 \%, p=0.83, n=12,12$; three rats $)$, or $\omega$-agatoxinIVA (100 nM) (Tocris Bioscience), a P-type VGCC blocker $(+9.45 \pm 8.58 \%, p=0.28, n=12,12$; three rats). These results are consistent with a model wherein secretin released from PCs regulates Kv1.2. However, the blockade of VGGCs could reduce GABA release, precluding an effect of gabazine on Kv1.2. To address this, we left calcium channel function intact, and directly inhibited the secretin receptor. We found gabazine had no effect on surface Kv1.2 in the presence of the secretin-receptor antagonist, $5-27$ secretin $(30 \mu \mathrm{M})(-2.36 \pm 6.66 \%, p=0.73, n=15,15$; three rats). A previous study has shown cerebellar secretin release occurs constitutively (Lee et al., 2005a). Given this, our model predicts that antagonizing the secretin receptor would elevate surface Kv1.2. Indeed, the secretin antagonist 5-27 secretin (30

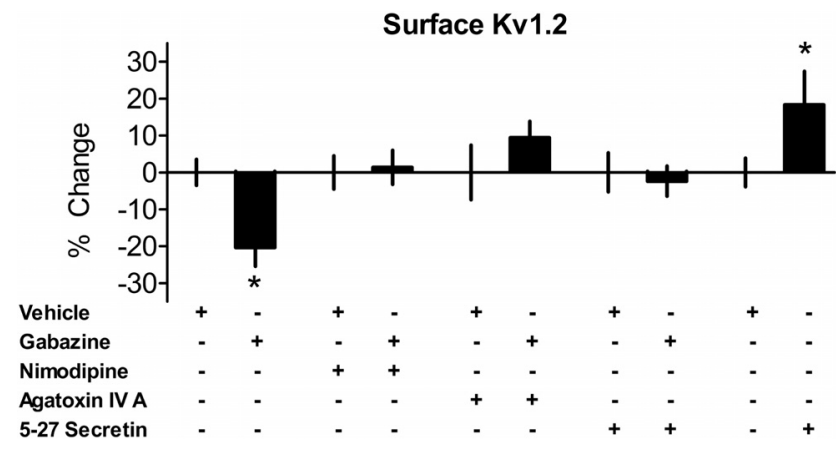

Figure 3. Endogenous secretin regulates cerebellar Kv1.2 surface expression. Change in mean surface Kv1.2 as determined by biotinylation and immunoblot of drug-treated cerebellar slices: a 45 min incubation with $\mathrm{GABA}_{\mathrm{A}}$ receptor antagonist, gabazine $(20 \mu \mathrm{M})$ reduces surface Kv1.2 ( $\left.{ }^{*} p \leq 0.003\right)$. The L-type calcium channel blocker nimodipine ( $20 \mu \mathrm{m} ; p \geq 0.83$ ) or the P-type calcium channel blocker $\omega$-agatoxin-IVA ( $100 \mathrm{~nm} ; p \geq 0.28$ ) blocks gabazine's effect on surface Kv1.2. Gabazine-induced reduction of surface Kv1.2 is also prevented by the secretin receptor antagonist, $5-27$ secretin, (30 $\mu \mathrm{m} ; p \geq 0.73)$. Antagonism of endogenous secretin with 5-27 secretin (30 $\mu \mathrm{m}$ for $15 \mathrm{~min}$ ) increases surface Kv1.2 relative to vehicle ( ${ }^{*} p \leq 0.05$ ).

$\mu \mathrm{M}, 15 \mathrm{~min})$, relative to vehicle, significantly elevated surface Kv1.2 ( $+18.35 \pm 9.82 \%, p=0.038, n=24,16$; three rats). Collectively, these findings support the hypothesis that endogenous secretin, like exogenous secretin, reduces surface Kv1.2.

\section{Identification of the locus of Kv1.2 trafficking in the cerebellar cortex}

$A C$-dependent Kv1.2 trafficking occurs in PC dendrites and $B C$ axon terminals

Detection of surface Kv1.2 by labeling with a cell-surface-specific marker. Kv1.2 is abundantly expressed in the molecular layer of the cerebellar cortex, particularly in PC dendrites (Sheng et al., 1994) where it affects excitatory input integration (Khavandgar et al., 2005) and may contribute to PC output (McKay et al., 2005). $\mathrm{Kv1.2}$ is also strongly expressed in the pinceau formation of BC axon terminals where it affects inhibitory synaptic transmission to PCs (Southan and Robertson, 1998a,b). Since endogenous secretin regulates Kv1.2, and since secretin acts via AC, we investigated whether AC regulates surface $\mathrm{Kv1.2}$ in PC dendrites, pinceaus of $\mathrm{BC}$ axon terminals, or both. To accomplish this, we developed a fluorescent dye-conjugated derivative of the relatively specific Kv1.2-specific toxin TsTx (Werkman et al., 1993; Rodrigues et al., 2003). We used a strategy similar to that developed to generate fluorescent conjugates of a less specific potassium channel inhibitory peptide, hongotoxin (Pragl et al., 2002). The linear peptide for TsTx was generated by Celtek Bioscience with a cysteine instead of the native alanine at site 20. Subsequently, the fluorescent dye ATTO 594 was conjugated to the engineered cysteine, and the toxin was refolded, purified, and validated by Alomone Labs.

After vehicle or forskolin treatment, cerebellar slices were fixed without permeabilization, and surface Kv1.2 was labeled with ATTO 594 TsTx (3 nM, overnight), rinsed, postfixed, and imaged by fluorescence microscopy. A positive signal was detected in the molecular layer in the territory of PC dendrites and in pinceau formations (Fig. $4 A$ ). We confirmed that slices fixed without permeabilization were grossly intact by the near absent labeling for the cytoplasmic $\mathrm{Kv} 1 \beta$-subunit $\mathrm{Kv} \beta 2$, compared with slices permeabilized with acetone after fixation (Fig. $4 I, J$ ).

AC stimulation with forskolin decreased the mean intensity of ATTO 594 signal in pinceau ROIs $(-27.56 \pm 4.33 \%, p \leq 0.0001$, $n=63,48$; four rats) (Fig. $4 B$ ). A similar analysis of ROIs within 

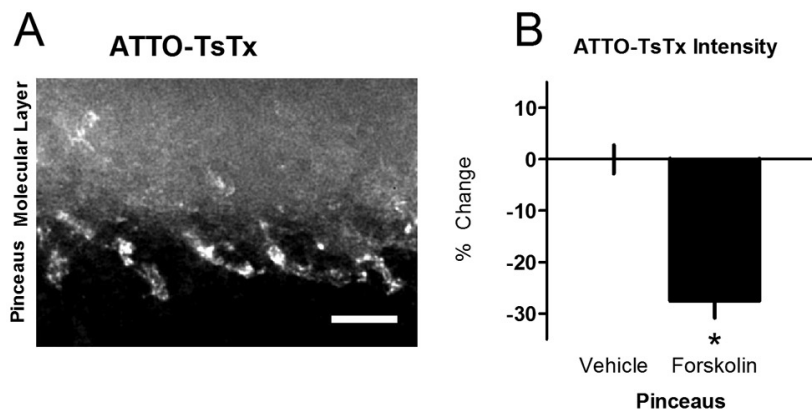

D
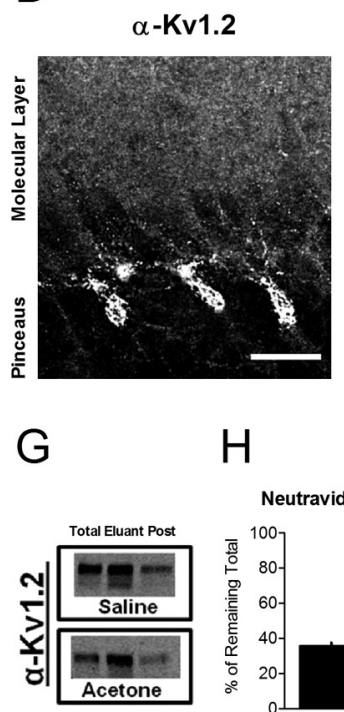

$\mathrm{H}$

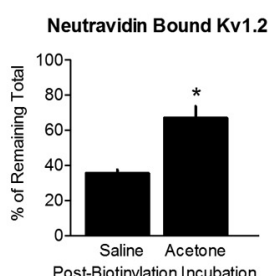

$\mathrm{K}$
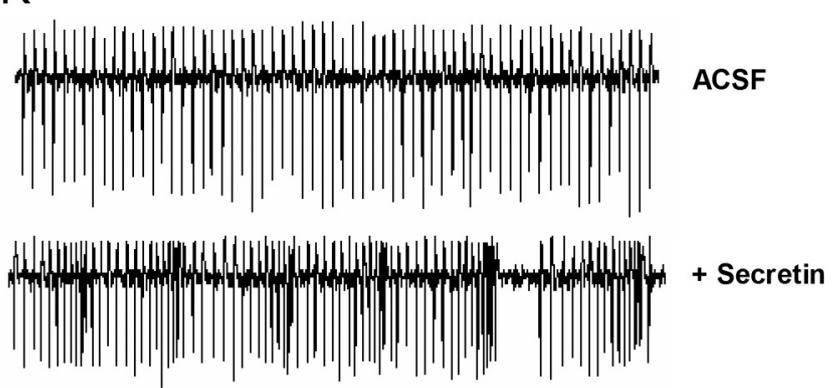

Figure 4. Stimulation of $A C$ reduces surface Kv1.2 at cerebellar pinceaus. $A, 0$ vernight incubation of fixed but not permeabilized rat cerebellar slices in ATT0-TsTX ( $3 \mathrm{~nm}$ ) gives positive signal in the molecular layer and in the pinceaus of BC axon terminals. Scale bar, $10 \mu \mathrm{m}$ $\boldsymbol{B}$, Mean intensity of ATT0-TsTx in pinceau ROls is lower in forskolin than in vehicle-treated slices $\left({ }^{*} p \leq 0.001\right)$. $\boldsymbol{C}$, In the same slices, the mean intensity of ATT0-TsTx in molecular layer R0ls is also reduced in forskolin versus vehicle-treated slices $\left({ }^{*} p \leq 0.005\right)$. D, $\alpha$-Kv1.2 staining in acetone extracted cerebellar slice to enrich for surface Kv1.2. Scale bar, $20 \mu \mathrm{m}$. $\boldsymbol{E}$, Forskolin significantly reduces the mean intensity of surface-enriched Kv1.2 in pinceau R0Is $\left({ }^{*} p \leq 0.0001\right)$. F, Forskolin also reduces the intensity of surface-enriched Kv1.2 in the molecular layer $\left({ }^{*} p \leq 0.05\right)$. G, Cerebellar slices are first biotinylated then incubated in saline or acetone. Kv1.2 is preferentially lost from the nonbiotinylatable internal population in the Post fraction after incubation of the Total lysate with neutravidin beads pulls out biotinylated surface Kv1.2, as seen in the Eluant. $\boldsymbol{H}$, Quantification of Western blots as in $\boldsymbol{G}$. Neutravidin removes more Kv1.2 from the lysates of slices that were incubated with acetone as opposed to saline after biotinylation ( ${ }^{*} p \leq 0.02$ ), indicating preferential extraction of nonsurface channel. I, J, Cytoplasmic $\alpha$-Kv $\beta 2$ staining thresholded to lgG control yields weak staining in the cortex of cerebellar slices without intentional permeabilization, demonstrating that cell membranes are grossly intact with the fixation protocols used for ATTO labeling as in $\boldsymbol{A}-\boldsymbol{C}$. Scale bars: $20 \mu \mathrm{m}$. $\boldsymbol{K}$, Single-cell extracellular action-potential recordings taken during ACSF perfusion (top) and after (bottom) addition of secretin show representative secretin-induced transient high-frequency bursts of action potentials in a tonically firing PC.

the molecular layer also revealed a significant decrease in ATTO 594 signal $(-22.9 \pm 7.77, p=0.0023, n=32,37$; four rats) (Fig. $4 C)$. Therefore, AC stimulation reduces surface Kv1.2 in both the $\mathrm{BC}$ axon terminals and in PC dendrites.
Detection of surface Kv1.2 by differential extraction with acetone

We next sought to support our finding that forskolin reduces surface Kv1.2 in the cerebellar cortex with a second assay. To do so we developed a method to preferentially extract nonsurface Kv1.2 relative to surface Kv1.2, thereby permitting detection of changes in surface channel levels using immunofluorescence. Biotinylation of surface proteins and quantification of the proportion of surface to total channel was performed as in the experiments for Figure 1, with the difference here that some slices were extracted with acetone after biotinylation of surface proteins. Biotinylated surface Kv1.2 is removed from the Total lysate by incubation with neutravidin beads, while nonsurface Kv1.2 remains in the post-neutravidin supernatant Post. Compared with slices incubated in saline, slices incubated in acetone after biotinylation showed proportionally less Kv1.2 in this nonsurface Post fraction (Fig. $4 G$ ). Quantifying this, we found that for slices not extracted with acetone, the surface proportion of total Kv1.2 was 35.39\% (similar to independent experiments shown in Fig. 1). In contrast, for slices extracted with acetone, the surface proportion of total Kv1.2 was $66.76 \%(+31.37 \pm 7.13 \%, p=$ $0.012, n=3$, 3; three rats) (Fig. $4 H$ ). If acetone had extracted both nonsurface and surface Kv1.2 equally, this value would have been the same between acetone-extracted and nonextracted slices. However, by preferentially extracting nonsurface Kv1.2, acetone enhances the representation of surface channel, facilitating detection of changes in surface Kv1.2 by immunofluorescence.

Using this method, we examined the effect of forskolin on surface Kv1.2 levels in the molecular layer and in pinceaus by immunofluorescence. As expected, a positive $\alpha$-Kv1.2 signal was observed in both regions (Fig. $4 D$ ). Furthermore, forskolin reduced the mean intensity of surfaceenriched Kv1.2 immunofluorescence in cerebellar pinceaus $(-17.3 \pm 3.58 \%, p \leq$ 0.0001, $n=99,102$; seven rats) (Fig. $4 E$ ), and in the molecular layer of the cerebellar cortex $(-16.02 \pm 8.69 \%, p=0.037$, $n=19,19$; seven rats) (Fig. $4 F$ ).

Thus, by two separate assays, we found that forskolin reduced surface Kv1.2 in the molecular layer and in $\mathrm{BC}$ axon terminal pinceaus. The functional significance of the decreased expression of Kv1.2 in pinceaus is indicated by published studies showing that, in rat cerebellar slices, secretin or forskolin (Yung et al., 2001) mimics the effect of pharmacological inhibition of Kv1.2, enhancing IPSC frequency recorded in PCs (Southan and Robertson, 1998a,b). In contrast, 
the effect of secretin on PC excitability was not known. Khavandgar et al. (2005) demonstrated that pharmacological inhibition of Kv1.2 causes an increase in PC dendritic excitability. Because secretin stimulates AC, and AC stimulation with forskolin decreased surface Kv1.2 in PC dendrites, it would be expected that secretin would mimic pharmacological inhibition of Kv1.2 to some degree, increasing dendritic excitability. We therefore tested the effect of secretin on two indices of enhanced dendritic excitability in PCs identified by Khavandgar et al. (2005): (1) the appearance of brief transient increases in action-potential frequency of PCs exhibiting the tonic firing pattern and (2) decreased cycle duration of PCs exhibiting the trimodal pattern of firing (Khavandgar et al., 2005). PC action-potential activity was recorded in the presence of 6-cyano-7-nitroquinoxaline-2,3dione $(100 \mu \mathrm{M})$ and gabazine $(10 \mu \mathrm{M})$ to block excitatory and inhibitory synaptic input, respectively, using single-cell extracellular recording from PC soma to prevent disruption of PC firing caused by whole-cell recording (Womack and Khodakhah, 2003). Secretin (30 nM) elicited an effect in four of seven cells tested, two of which started in a trimodal pattern of cyclic firing, and two of which started in a tonic firing pattern. A trimodal pattern of cyclic firing is defined as including tonic, bursting, and quiescent phases. The number of such cycles recorded in a $5 \mathrm{~min}$ period before secretin addition, and in the 5 min period starting 10 min after secretin application, were 2:8 and 3:8 in each, respectively. In the two cells that exhibited tonic firing, secretin elicited the appearance of high-frequency bursts with the following characteristics for cell 1 and cell 2 , respectively: frequency $=215$ and $228 \mathrm{~Hz}$, burst duration $=48$ and $32 \mathrm{~ms}$, and 14 and 9 events per burst. The baseline firing rate was unchanged by addition of secretin (cell $1=48.2$ vs $48.4 \mathrm{~Hz}$; cell $2=102$ vs $100 \mathrm{~Hz}$ ). Thus, in four of seven cells, secretin affected PC firing patterns in a manner consistent with it having enhanced dendritic excitability. Interestingly, two of the three cells that did not respond to secretin exhibited transient high-frequency action-potential bursts during the pre-secretin baseline recording period, suggesting that in these cells Kv1.2 may have already been at least partially suppressed.

\section{Kv1.2 function in cerebellar cortex affects cerebellum- dependent learning in rats}

Intracerebellar cortex infusion of either a Kv1.2 inhibitor (TsTx) or secretin enhances $E B C$

The AC-induced reduction of surface Kv1.2 in pinceaus likely causes an increase in inhibition to the PCs (Xie et al., 2010). PC inhibition has been demonstrated to be important for adaptation of the vestibulo-ocular reflex, a form of cerebellar-dependent learning (Wulff et al., 2009). This suggests that suppression of Kv1.2 function might be an endogenous mechanism for regulating learned behaviors controlled by the cerebellum. EBC is a well studied form of cerebellum-dependent learning for which the entire brainstem-cerebellar circuit has been identified (Thompson, 1986; Medina et al., 2000; De Zeeuw and Yeo, 2005; Thompson and Steinmetz, 2009; Freeman and Steinmetz, 2011). Briefly, the tone CS is relayed through the pontine nuclei to the cerebellar cortex via mossy fiber (MF) projections to cerebellar granule cells (GCs) and to one of the deep cerebellar nuclei, the interpositus nucleus (IP), via MF collaterals. Tone CS information reaches PCs via parallel fiber (PF) axons from GCs. The periorbital stimulation US is relayed through the inferior olive to the cerebellar cortex via climbing fiber (CF) projections to PCs and to the IP via CF collaterals. PCs tonically inhibit the deep cerebellar nuclei. A standard model has been that EBC leads to plastic changes in
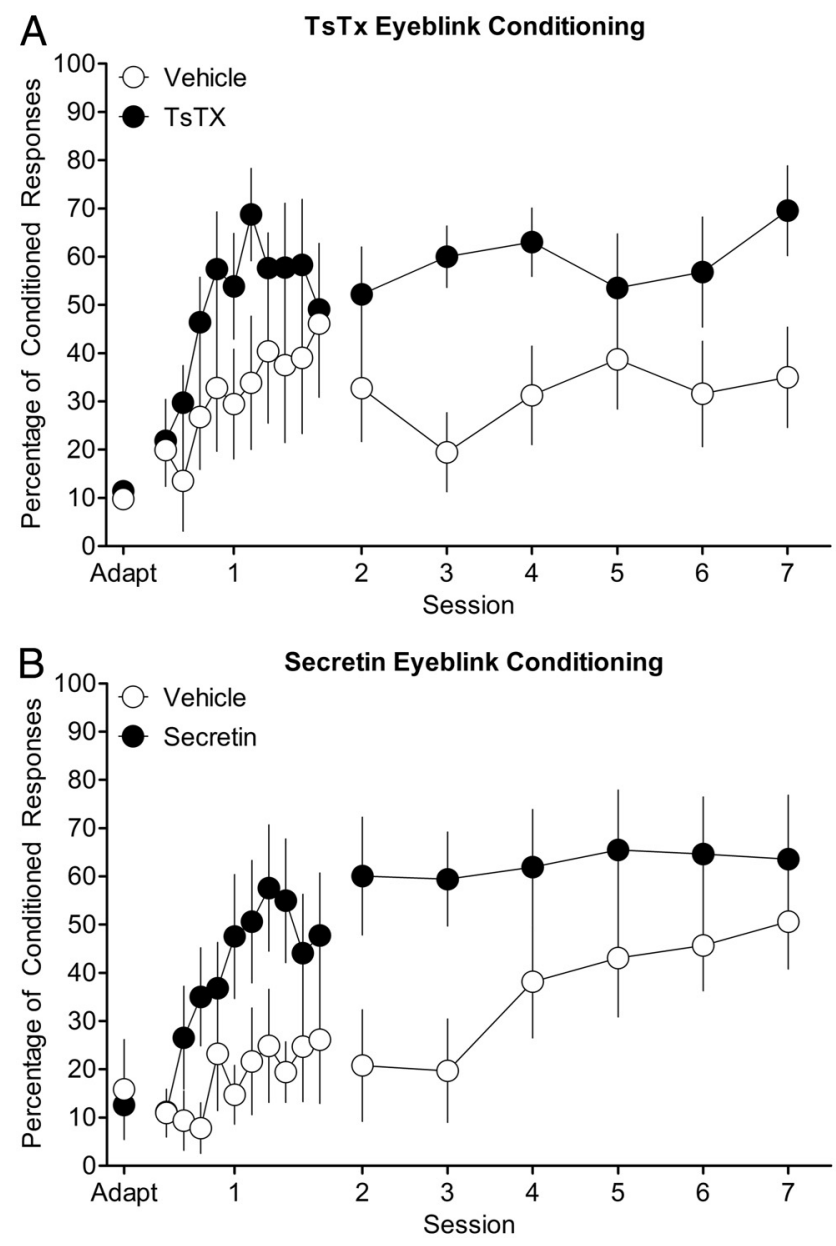

Figure 5. Secretin and Kv1.2 inhibition with TsTx similarly affect cerebellum-dependent learning. $A, B$, The percentage of CRs on CS-US trials. Error bars indicate SEM. $A$, TSTX, versus vehicle infusion, into lobulus simplex of rat cerebellar cortex enhances EBC. A 2 (Group) $\times 7$ (Session) repeated-measures ANOVA on the percentage of CRs on CS-US trials revealed a significant main effect of Group, $F_{(1,12)}=5.41, p<0.04$. In Session 1, on a block-by-block basis where each block consisted of 8 CS-US trials, a 2 (Group) $\times 10$ (Block) repeated-measures ANOVA on percentage of $C R s$ on CS-US trials revealed a significant main effect of Block, $F_{(9,108)}$ $=4.71, p<0.01$ but no difference between groups. $B$, Secretin, versus vehicle, infusion into lobulus simplex of rat cerebellar cortex enhances EBC. A 2 (Group) $\times 7$ (Session) repeatedmeasures ANOVA on the percentage of CRs on CS-US trials revealed a significant main effect of Session, $F_{(6,84)}=12.61, p<0.01$ and a significant Group $\times$ Session interaction effect, $F_{(6,84)}$ $=3.03, p<0.01$. In Session 1 , on a block-by-block basis where each block consisted of eight CS-US trials, a 2 (Group) $\times 10$ (Block) repeated-measures ANOVA on the percentage of CRs on CS-US trials revealed a significant main effect of Block, $F_{(9,126)}=4.45, p<0.01$, but no difference between groups.

cerebellar cortex that disinhibit the IP and allow the eyeblink CR to be generated in response to the tone CS. Given the fact that BCs are in a position to regulate $\mathrm{PCs}, \mathrm{BCs}$ are positioned to play a critical role in EBC. In this model, suppression of Kv1.2 function in BCs, by enhancing GABA release, would inhibit PCs, thereby disinhibiting the IP and facilitating EBC by permitting MF-IP plasticity to occur more quickly.

We therefore tested whether suppression of Kv1.2 would enhance cerebellar-dependent learning. In the first experiment, we infused, via a prepositioned cannula, the Kv1.2 inhibitor TsTx into lobulus simplex of cerebellar cortex before EBC sessions. All cannula tips were located in lobulus simplex ipsilateral to the eye that received the US. Rats that received TsTx infusions into lobulus simplex immediately before Sessions 1-6 of EBC learned faster than vehicle rats (Fig. 5A). These observations were con- 
firmed by a 2 (Group) $\times 7$ (Session) repeated-measures ANOVA on the percentage of CRs on CS-US trials, which revealed a significant main effect of Group, $F_{(1,12)}=5.41, p<0.04$. We further analyzed performance in Session 1 on a block-by-block basis, where each block consisted of 8 CS-US trials. A 2 (Group) $\times 10$ (Block) repeated-measures ANOVA on percentage of CRs on CS-US trials revealed a significant main effect of Block, $F_{(9,108)}=$ $4.71, p<0.01$, but no difference between groups. A series of 2 (Group) $\times 7$ (Session) repeated-measures ANOVAs on CR onset latency, CR amplitude, and UR amplitude did not reveal any differences between groups ( $p$ values $>0.13)$. A 2 (Group) $\times 7$ (Session) repeated-measures ANOVA on percentage of SRs revealed a significant main effect of Session, $F_{(6,72)}=2.80, p<0.02$ and a marginally significant main effect of Group, $F_{(1,12)}=4.56$, $p=0.054$. However, percentage of trials with an SR was very low $(<3 \%)$ in both groups across sessions.

The finding that pharmacological inhibition of Kv1.2 enhances EBC suggested that inhibition of Kv1.2 via a more physiological mechanism would have a similar effect. To test this idea, we infused secretin into the lobulus simplex and assessed its effects on EBC. Rats that received secretin infusions into lobulus simplex immediately before Sessions 1-3 of EBC learned faster than vehicle rats (Fig. $5 B$ ). These observations were confirmed by a 2 (Group) $\times 7$ (Session) repeated-measures ANOVA on the percentage of CRs on CS-US trials, which revealed a significant main effect of Session, $F_{(6,84)}=12.61, p<0.01$ and a significant Group $\times$ Session interaction effect, $F_{(6,84)}=3.03, p<0.01$. Post hoc tests of the significant interaction effect with a series of oneway ANOVAs revealed that secretin-infused rats displayed a significantly greater percentage of CRs in Sessions $2(p=0.016)$ and $3(p=0.006)$ of conditioning. We further analyzed performance in Session 1 on a block-by-block basis, where each block consisted of eight CS-US trials. A 2 (Group) $\times 10$ (Block) repeatedmeasures ANOVA on the percentage of CRs on CS-US trials revealed a significant main effect of Block, $F_{(9,126)}=4.45, p<$ 0.01 but no difference between groups. A series of 2 (Group) $\times 7$ (Session) repeated-measures ANOVAs on CR onset latency, UR amplitude, and the percentage of SRs failed to reveal any differences between groups ( $p$ values $>0.10)$. A 2 (Group) $\times 7$ (Session) repeated-measures ANOVA on CR amplitude revealed a significant main effect of Session, $F_{(6,84)}=3.88, p<0.01$ and a significant Group $\times$ Session interaction effect, $F_{(6,84)}=3.12, p<$ 0.01 . Post hoc tests of the significant interaction effect with a series of one-way ANOVAs failed to reveal any significant differences between Groups in any Session ( $p$ 'values $>0.09$ ).

\section{Discussion}

The voltage-gated potassium channel Kv1.2 modulates excitatory and inhibitory synaptic input to PCs (Southan and Robertson, 1998a,b, 2000; Tan and Llano, 1999). In this study we provide the first evidence that cerebellar Kv1.2 is regulated by endocytic trafficking, that this process is modulated by AC, and that this channel regulation may have significance as a component of cerebellumdependent learning.

In Figures 1-3 we used biotinylation of cell-surface proteins to demonstrate that cerebellar Kv1.2 is regulated by AC-dependent endocytic trafficking. While this approach is powerful because it detects changes in Kv1.2 trafficking throughout an entire slice, it does not provide information about which population of Kv1.2, $\mathrm{BC}$ axon terminal or PC dendritic, is sensitive to AC-mediated trafficking. It was important to investigate the location of the AC-modulated pool of surface Kv1.2 because suppression of
Kv1.2 at each site would likely have distinct effects on PC activity and cerebellar function.

Using two independent microscopy techniques, we found that stimulating AC decreased surface Kv1.2 within pinceaus (Fig. 4). The physiological role of this decrease is suggested by previous studies that examined the effect of secretin or pharmacological block of Kv1.2 on inhibitory input to PCs. Forskolin and secretin cause an AC-dependent increase in IPSC frequency and amplitude recorded in PCs (Yung et al., 2001). Pharmacological suppression of Kv1.2 also increases inhibitory input to PCs (Southan and Robertson, 1998a,b), suggesting a link between secretin and Kv1.2 effects on PC inhibition. Our data, in conjunction with a previous study showing that Kv1.2 endocytosis suppresses Kv1.2 ionic current (Nesti et al., 2004), suggests a mechanism for this link whereby secretin stimulation of AC increases channel endocytosis. It is important to note, however, that the increase in inhibitory input to PCs caused by secretin involves an ACindependent component (Yung et al., 2001), which would not be expected to involve Kv1.2 since the AC-inhibitor SQ22536 blocks the ability of secretin to modulate surface Kv1.2 levels.

The stimulation of AC by forskolin also potentiates excitatory transmission at the PF to PC synapse and multiple lines of evidence point to a presynaptic mechanism (Salin et al., 1996; Chen and Regehr, 1997). However, the PF:PC synapse is also governed by a postsynaptic mechanism involving Kv1.2. Inhibition of Kv1.2 within the molecular layer increases dendritic excitability and enhances PF:PC-evoked postsynaptic currents (Khavandgar et al., 2005). A presynaptic role for Kv1.2 in this phenomenon seems unlikely since no effect was observed on presynaptic calcium. Rather, Kv1.2 blockade appears to enhance PF:PC-evoked currents by increasing dendritic excitability directly (Khavandgar et al., 2005). Consistent with this, we show AC stimulation with secretin also increased dendritic excitability (Fig. 5), and decreased surface Kv1.2 levels in the molecular layer (Fig. 4). Thus, AC-dependent potentiation of the PF:PC synapse could include a postsynaptic mechanism.

The data in Figures 1-4 identifying cellular mechanisms for secretin-mediated suppression of Kv1.2 in BCs and PCs raises the question of whether this regulation impacts cerebellumdependent behavior. The data in Figure $5 A$, showing that infusion of TsTx into the cerebellar cortex enhanced EBC, indicates inhibition of Kv1.2 does indeed affect cerebellum-dependent behavior. Our finding that secretin infusion mimicked this behavioral effect (Fig. $5 B$ ) suggests that suppression of Kv1.2 by endogenous secretin, as shown in Figure 3, could be a mechanism for regulating cerebellar EBC. Our infusions likely influenced both BCs and PCs, given the presence of secretin receptors (Yung et al., 2001) and Kv1.2 (Chung et al., 2001) in both cell types. However, it is important to note that, as detailed below, the finding that EBC was enhanced rather than impaired suggests that inhibition of PCs may have dominated at the systems level. Indeed, our behavioral experiments were designed with this prediction in mind, using a long-delay procedure to enhance resolution of potential facilitation of EBC.

The synaptic mechanisms of plasticity in cerebellar cortex that underlie EBC have remained somewhat elusive. PF:PC long-term depression (LTD) has been the most studied candidate. On one hand, mutant mice with deficient PF:PC LTD induction due to lack of mGluR1 (Aiba et al., 1994; Kishimoto et al., 2001) or GluR $\delta 2$ (Kishimoto et al., 2001; Kakegawa et al., 2008) in PCs show poor EBC. This is consistent with the necessity for removal of PC inhibition of the IP for EBC to proceed normally. On the other hand, there are data that call into question the strength of 
the relationship between PF:PC LTD and EBC. For example, mutant mice with enhanced, rather than impaired, PF:PC LTD also show deficient EBC (Koekkoek et al., 2005), pharmacological disruption of LTD in rats does not affect EBC (Welsh et al., 2005), and a study of three different strains of mutant mice with deficient cerebellar LTD showed these mice conditioned normally (Schonewille et al., 2011). Thus, PF:PC LTD appears to be only part of the mechanisms underlying EBC.

Our results lead us to propose that a new mechanism of cerebellar cortical synaptic plasticity important in EBC may involve secretindependent suppression of Kv1.2. During EBC, converging CS (MF/ $\mathrm{PF}$ ) and US (CF) inputs to a subset of PCs would be expected to depolarize these cells and thereby enhance their release of secretin (Lee et al., 2005a). Once released, secretin might act both as a retrograde transmitter to regulate $\mathrm{Kv} 1.2$ in the $\mathrm{BC}$ axon terminal and as an autocrine/paracrine signal to regulate Kv1.2 in PC dendrites (Yung et al., 2001; Ng et al., 2002; Lee et al., 2005a).

Secretin, acting as a retrograde messenger, could activate receptors on $\mathrm{BC}$ axon terminals to stimulate $\mathrm{AC}$-dependent endocytic trafficking of Kv1.2. This loss of surface Kv1.2 is hypothesized to reduce Kv1.2 ionic current, enhancing GABA release and thus inhibition of these same PCs (Southan and Robertson, 1998a,b). This mechanism may partially counter the LTD of inhibitory inputs to PCs that is also induced by CF input (Mittmann and Häusser, 2007), which would presumably occur during EBC. LTD of inhibitory inputs opposes the increase in inhibition produced by CF-driven LTD at PF:PC synapses (Mittmann and Häusser, 2007). Thus, it is possible that regulation of Kv1.2 at BC terminals contributes, along with other mechanisms (Bao et al., 2010), to ensure that inhibition of the PCs activated by CF input eventually dominates. This inhibition of PCs would relieve inhibition of select deep cerebellar nuclei neurons in the IP, allowing MF-IP synapses to strengthen more quickly, enhancing EBC.

Secretin, acting as an autocrine/paracrine signal, could also enhance EBC. A previous report suggested that Kv1.2 opposes dendritic calcium influx and therefore the induction of LTD (Khavandgar et al., 2005). The regional reduction of dendritic Kv1.2 function by secretin-stimulated channel endocytosis could enhance PF:PC LTD by increasing sensitivity of specific PF synaptic inputs during coincident CF inputs. Under this model, dendritic Kv1.2 suppression would have the same net effect as BC axon terminal Kv1.2 suppression: reducing PC output and disinhibiting the IP.

Our data do not inform whether secretin facilitates EBC through facilitation of PF:PC LTD, by elevation of PC inhibition, both means, or by alternate mechanisms. However, the proposed models give plausible mechanisms by which the regulation of Kv1.2 may be an important contributing factor. Determining the mechanisms behind how secretin enhances cerebellar-dependent learning is of interest, especially since, paralleling our animal studies, in studies of patients with schizophrenia secretin also enhances EBC (Bolbecker et al., 2009).

In this study we focused on secretin receptor-mediated regulation of Kv1.2. However, the cerebellum contains a variety of other receptors likely to regulate $\mathrm{Kv1.2}$. One logical candidate is the $\mathrm{Gi}$-coupled $\mathrm{CB} 1$ receptor. $\mathrm{CB} 1$ receptors are expressed in $\mathrm{BC}$ axon terminals, where their activation reduces inhibition of PCs (Szabo et al., 2004). One mechanism may be CB1 receptormediated inhibition of $\mathrm{AC}$ and a resulting increased surface expression of presynaptic Kv1.2. Further, like secretin, endocannabinoids are thought to be released from depolarized PCs (Yoshida et al., 2002). Because secretin and cannabinoids would have opposing effects on surface Kv1.2 in BC axon terminals, PCs might regulate inhibition they receive by altering the ratio of secretin and cannabinoids they release. It is interesting to speculate this ratio could be determined by changes in PC firing patterns.

Kv1.2 may also be regulated by other receptors expressed in $\mathrm{BC}$ axon terminals or PC dendrites, including c-Kit receptor tyrosine kinase (Manova et al., 1992; Kim et al., 2003), metabotropic glutamate (Neale et al., 2001a,b), NMDA (Glitsch and Marty, 1999), metabotropic GABA (Batchelor and Garthwaite, 1992), purinergic (Kocsis et al., 1984; Wall et al., 2007), or adrenergic receptors (Saitow et al., 2000; Hirono and Obata, 2006).

Further regulatory diversity could result from differences in $\alpha$ - and $\beta$-subunit composition of Kv1.2 containing holochannels, which might differ between BCs and PCs (Koch et al., 1997). For example, Kv1.2 in BC axon terminals consists mostly of Kv1.2/Kv1.1 heteromultimers (Koch et al., 1997). Less is known about the composition of PC Kv1.2-containing channels; however, PCs express Kv1.5, suggesting the existence of Kv1.2/Kv1.5 heteromultimers in these cells (Chung et al., 2001).

\section{References}

Aiba A, Kano M, Chen C, Stanton ME, Fox GD, Herrup K, Zwingman TA, Tonegawa S (1994) Deficient cerebellar long-term depression and impaired motor learning in mGluR1 mutant mice. Cell 79:377-388.

Bao J, Reim K, Sakaba T (2010) Target-dependent feedforward inhibition mediated by short-term synaptic plasticity in the cerebellum. J Neurosci 30:8171-8179.

Batchelor AM, Garthwaite J (1992) GABAB Receptors in the Parallel Fibre Pathway of Rat Cerebellum. Eur J Neurosci 4:1059-1064.

Bolbecker AR, Hetrick WP, Johannesen JK, O'Donnell BF, Steinmetz JE, Shekhar AS (2009) Secretin effects on cerebellar-dependent motor learning in schizophrenia. Am J Psychiatry 166:460-466.

Chen C, Regehr WG (1997) The mechanism of cAMP-mediated enhancement at a cerebellar synapse. J Neurosci 17:8687-8694.

Chung YH, Shin C, Kim MJ, Lee BK, Cha CI (2001) Immunohistochemical study on the distribution of six members of the Kvl channel subunits in the rat cerebellum. Brain Res 895:173-177.

Connors EC, Ballif BA, Morielli AD (2008) Homeostatic regulation of Kv1.2 potassium channel trafficking by cyclic AMP. J Biol Chem 283:3445-3453.

De Simoni A, Yu LM (2006) Preparation of organotypic hippocampal slice cultures: interface method. Nat Protoc 1:1439-1445.

De Zeeuw CI, Yeo CH (2005) Time and tide in cerebellar memory formation. Curr Opin Neurobiol 15:667-674.

Freeman JH, Steinmetz AB (2011) Neural circuitry and plasticity mechanisms underlying delay eyeblink conditioning. Learn Mem 18:666-677.

Gähwiler BH (1975) The effects of GABA, Picrotoxin and bicuculline on the spontaneous bioelectric activity of cultured cerebellar Purkinje cells. Brain Res 99:85-95.

Glitsch M, Marty A (1999) Presynaptic effects of NMDA in cerebellar Purkinje cells and interneurons. J Neurosci 19:511-519.

Hirono M, Obata K (2006) Alpha-adrenoceptive dual modulation of inhibitory GABAergic inputs to Purkinje cells in the mouse cerebellum. J Neurophysiol 95:700-708.

Kakegawa W, Miyazaki T, Emi K, Matsuda K, Kohda K, Motohashi J, Mishina M, Kawahara S, Watanabe M, Yuzaki M (2008) Differential regulation of synaptic plasticity and cerebellar motor learning by the C-terminal PDZ-binding motif of GluRdelta2. J Neurosci 28:1460-1468.

Khavandgar S, Walter JT, Sageser K, Khodakhah K (2005) Kv1 channels selectively prevent dendritic hyperexcitability in rat Purkinje cells. J Physiol 569:545-557.

Kim D, Im JO, Won YJ, Yoon SY, Lee EJ, Lee JH, Hong HN (2003) Upregulation of c-Kit receptor and stem cell factor in cerebellar inhibitory synapses in response to kainic acid. J Neurosci Res 71:72-78.

Kishimoto Y, Kawahara S, Fujimichi R, Mori H, Mishina M, Kirino Y (2001) Impairment of eyeblink conditioning in GluRdelta2-mutant mice depends on the temporal overlap between conditioned and unconditioned stimuli. Eur J Neurosci 14:1515-1521. 
Koch RO, Wanner SG, Koschak A, Hanner M, Schwarzer C, Kaczorowski GJ, Slaughter RS, Garcia ML, Knaus HG (1997) Complex subunit assembly of neuronal voltage-gated $\mathrm{K}+$ channels. Basis for high-affinity toxin interactions and pharmacology. J Biol Chem 272:27577-27581.

Kocsis JD, Eng DL, Bhisitkul RB (1984) Adenosine selectively blocks parallel-fiber-mediated synaptic potentials in rat cerebellar cortex. Proc Natl Acad Sci U S A 81:6531-6534.

Koekkoek SK, et al. (2005) Deletion of FMR1 in Purkinje cells enhances parallel fiber LTD, enlarges spines, and attenuates cerebellar eyelid conditioning in Fragile X syndrome. Neuron 47:339-352.

Köves K, Kausz M, Reser D, Horváth K (2002) What may be the anatomical basis that secretin can improve the mental functions in autism? Regul Pept 109:167-172.

Köves K, Kausz M, Reser D, Illyes G, Takács J, Heinzlmann A, Gyenge E, Horváth K (2004) Secretin and autism: a basic morphological study about the distribution of secretin in the nervous system. Regul Pept 123:209-216.

Laube G, Röper J, Pitt JC, Sewing S, Kistner U, Garner CC, Pongs O, Veh RW (1996) Ultrastructural localization of Shaker-related potassium channel subunits and synapse-associated protein 90 to septate-like junctions in rat cerebellar pinceaux. Brain Res Mol Brain Res 42:51-61.

Lee SM, Chen L, Chow BK, Yung WH (2005a) Endogenous release and multiple actions of secretin in the rat cerebellum. Neuroscience 134:377-386.

Lee SM, Yung WH, Chen L, Chow BK (2005b) Expression and spatial distribution of secretin and secretin receptor in human cerebellum. Neuroreport 16:219-222.

Manova K, Bachvarova RF, Huang EJ, Sanchez S, Pronovost SM, Velazquez E, McGuire B, Besmer P (1992) c-kit receptor and ligand expression in postnatal development of the mouse cerebellum suggests a function for c-kit in inhibitory interneurons. J Neurosci 12:4663-4676.

McKay BE, Molineux ML, Mehaffey WH, Turner RW (2005) Kv1 K+ channels control Purkinje cell output to facilitate postsynaptic rebound discharge in deep cerebellar neurons. J Neurosci 25:1481-1492.

Medina JF, Nores WL, Ohyama T, Mauk MD (2000) Mechanisms of cerebellar learning suggested by eyelid conditioning. Curr Opin Neurobiol 10:717-724.

Mittmann W, Häusser M (2007) Linking synaptic plasticity and spike output at excitatory and inhibitory synapses onto cerebellar Purkinje cells. J Neurosci 27:5559-5570.

Moyer JR Jr, Brown TH (1998) Methods for whole-cell recording from visually preselected neurons of perirhinal cortex in brain slices from young and aging rats. J Neurosci Methods 86:35-54.

Neale SA, Garthwaite J, Batchelor AM (2001a) Metabotropic glutamate receptor subtypes modulating neurotransmission at parallel fibre-Purkinje cell synapses in rat cerebellum. Neuropharmacology 41:42-49.

Neale SA, Garthwaite J, Batchelor AM (2001b) mGlul receptors mediate a post-tetanic depression at parallel fibre-Purkinje cell synapses in rat cerebellum. Eur J Neurosci 14:1313-1319.

Nesti E, Everill B, Morielli AD (2004) Endocytosis as a mechanism for tyrosine kinase-dependent suppression of a voltage-gated potassium channel. Mol Biol Cell 15:4073-4088.

Ng SS, Yung WH, Chow BK (2002) Secretin as a neuropeptide. Mol Neurobiol 26:97-107.

Pragl B, Koschak A, Trieb M, Obermair G, Kaufmann WA, Gerster U, Blanc E, Hahn C, Prinz H, Schütz G, Darbon H, Gruber HJ, Knaus HG (2002) Synthesis, characterization, and application of cy-dye- and alexa-dyelabeled hongotoxin(1) analogues. The first high affinity fluorescence probes for voltage-gated $\mathrm{K}+$ channels. Bioconjug Chem 13:416-425.

Rodrigues AR, Arantes EC, Monje F, Stühmer W, Varanda WA (2003) Tityustoxin-K(alpha) blockade of the voltage-gated potassium channel Kv1.3. Br J Pharmacol 139:1180-1186.

Ruxton GD (2006) The unequal variance t-test is an underused alternative to Student's t-test and the Mann-Whitney U test. Behav Ecol 17:688-690.

Saitow F, Satake S, Yamada J, Konishi S (2000) beta-adrenergic receptormediated presynaptic facilitation of inhibitory GABAergic transmission at cerebellar interneuron-Purkinje cell synapses. J Neurophysiol 84:2016-2025.

Salin PA, Malenka RC, Nicoll RA (1996) Cyclic AMP mediates a presynaptic form of LTP at cerebellar parallel fiber synapses. Neuron 16:797-803.

Schonewille M, Gao Z, Boele HJ, Veloz MF, Amerika WE, Simek AA, De Jeu MT, Steinberg JP, Takamiya K, Hoebeek FE, Linden DJ, Huganir RL, De Zeeuw CI (2011) Reevaluating the role of LTD in cerebellar motor learning. Neuron 70:43-50.
Sheng M, Tsaur ML, Jan YN, Jan LY (1994) Contrasting subcellular localization of the Kv1.2 K+ channel subunit in different neuron of rat brain. J Neurosci 14:2408-2417.

Siu FK, Lam IP, Chu JY, Chow BK (2006) Signaling mechanisms of secretin receptor. Regul Pept 137:95-104.

Southan AP, Robertson B (1998a) Patch-clamp recordings from cerebellar basket cell bodies and their presynaptic terminals reveal an asymmetric distribution of voltage-gated potassium channels. J Neurosci 18:948-955.

Southan AP, Robertson B (1998b) Modulation of inhibitory post-synaptic currents (IPSCs) in mouse cerebellar Purkinje and basket cells by snake and scorpion toxin $\mathrm{K}+$ channel blockers. Br J Pharmacol 125:1375-1381.

Southan AP, Robertson B (2000) Electrophysiological characterization of voltage-gated $\mathrm{K}(+)$ currents in cerebellar basket and Purkinje cells: Kv1 and Kv3 channel subfamilies are present in basket cell nerve terminals. J Neurosci 20:114-122.

Stirling L, Williams MR, Morielli AD (2009) Dual roles for RHOA/RHOkinase in the regulated trafficking of a voltage-sensitive potassium channel. Mol Biol Cell 20:2991-3002.

Szabo B, Than M, Thorn D, Wallmichrath I (2004) Analysis of the effects of cannabinoids on synaptic transmission between basket and Purkinje cells in the cerebellar cortex of the rat. J Pharmacol Exp Ther 310:915-925.

Tan YP, Llano I (1999) Modulation by K+ channels of action potentialevoked intracellular $\mathrm{Ca} 2+$ concentration rises in rat cerebellar basket cell axons. J Physiol 5201:65-78.

Thompson RF (1986) The neurobiology of learning and memory. Science 233:941-947.

Thompson RF, Steinmetz JE (2009) The role of the cerebellum in classical conditioning of discrete behavioral responses. Neuroscience 162:732-755.

Wall MJ, Atterbury A, Dale N (2007) Control of basal extracellular adenosine concentration in rat cerebellum. J Physiol 582:137-151.

Wang H, Kunkel DD, Schwartzkroin PA, Tempel BL (1994) Localization of Kv1.1 and Kv1.2, two K channel proteins, to synaptic terminals, somata, and dendrites in the mouse brain. J Neurosci 14:4588-4599.

Welsh JP, Yamaguchi H, Zeng XH, Kojo M, Nakada Y, Takagi A, Sugimori M, Llinás RR (2005) Normal motor learning during pharmacological prevention of Purkinje cell long-term depression. Proc Natl Acad Sci U S A 102:17166-17171.

Werkman TR, Gustafson TA, Rogowski RS, Blaustein MP, Rogawski MA (1993) Tityustoxin-K alpha, a structurally novel and highly potent K+ channel peptide toxin, interacts with the alpha-dendrotoxin binding site on the cloned Kv1.2 K+ channel. Mol Pharmacol 44:430-436.

Wessels-Reiker M, Basiboina R, Howlett AC, Strong R (1993) Vasoactive intestinal polypeptide-related peptides modulate tyrosine hydroxylase gene expression in PC12 cells through multiple adenylate cyclase-coupled receptors. J Neurochem 60:1018-1029.

Williams MR, Markey JC, Doczi MA, Morielli AD (2007) An essential role for cortactin in the modulation of the potassium channel Kv1.2. Proc Natl Acad Sci U S A 104:17412-17417.

Womack MD, Khodakhah K (2003) Somatic and dendritic smallconductance calcium-activated potassium channels regulate the output of cerebellar Purkinje neurons. J Neurosci 23:2600-2607.

Wulff $\mathrm{P}$, Schonewille M, Renzi M, Viltono L, Sassoè-Pognetto M, Badura A, Gao Z, Hoebeek FE, van Dorp S, Wisden W, Farrant M, De Zeeuw CI (2009) Synaptic inhibition of Purkinje cells mediates consolidation of vestibulo-cerebellar motor learning. Nat Neurosci 12:1042-1049.

Xie G, Harrison J, Clapcote SJ, Huang Y, Zhang JY, Wang LY, Roder JC (2010) A new Kv1.2 channelopathy underlying cerebellar ataxia. J Biol Chem 285:32160-32173.

Yang XL, Huang YZ, Xiong WC, Mei L (2005) Neuregulin-induced expression of the acetylcholine receptor requires endocytosis of ErbB receptors. Mol Cell Neurosci 28:335-346.

Yoshida T, Hashimoto K, Zimmer A, Maejima T, Araishi K, Kano M (2002) The cannabinoid $\mathrm{CB} 1$ receptor mediates retrograde signals for depolarization-induced suppression of inhibition in cerebellar Purkinje cells. J Neurosci 22:1690-1697.

Yuan Y, Lee LT, Ng SS, Chow BK (2011) Extragastrointestinal functions and transcriptional regulation of secretin and secretin receptors. Ann N Y Acad Sci 1220:23-33.

Yung WH, Leung PS, Ng SS, Zhang J, Chan SC, Chow BK (2001) Secretin facilitates GABA transmission in the cerebellum. J Neurosci 21:70637068 . 